\title{
Presencia de Cyperus papyrus L. (Cyperaceae) en la región biogeográfica atlántica de la Península Ibérica
}

\section{Rafael Carballeira' ${ }^{1}$ \& Martín Souto ${ }^{2}$}

${ }^{1}$ Centro de Investigacións Cientificas Avanzadas (CICA). Facultade de Ciencias, Universidade da Coruña, 15071 A Coruña (Galicia, España)

${ }^{2}$ Museo de Historia Natural, Universidad de Santiago de Compostela. Parque Vista Alegre, 15705 Santiago de Compostela (Galicia, España)

\author{
Correspondencia \\ Rafael Carballeira \\ e-mail: r.carballeira@udc.es \\ Recibido: 1 julio 2018 \\ Aceptado: 28 septiembre 2018 \\ Publicado on-line: octubre 2018
}

\begin{abstract}
Presence of Cyperus papyrus L. (Cyperaceae) in the Atlantic biogeographical region of the Iberian Peninsula
\end{abstract}

Palabras clave: Especie alóctona, Cyperus papyrus, ecología, distribución, interés cultural, Península Ibérica.

Key words: Non-indigenous species, Cyperus papyrus, ecology, distribution, cultural heritage, Iberian Peninsula.
Cyperus papyrus L. (papiro) es un helófito nativo de los humedales de África conocido desde la antigüedad en la cuenca del río Nilo, y ampliamente cultivada como planta ornamental a lo largo de la historia. Esta condición ha posibilitado su cultivo e introducción en el medio natural en áreas geográficas dispares y distantes a lo largo del mundo con el ser humano como vector (Allen, 1996; Verloove, 2014; Kipkemboi \& van Dam, 2016, Beentje, 2017). Su distribución actual como xenófito está ampliamente extendida, principalmente en áreas geográficas de climas cálidos de América y Asia, tan solo llegando a ser considerada como una especie invasora en Colombia (Ramírez et al., 2012). En Europa la presencia de la especie se da desde antiguo, pero las poblaciones naturalizadas son escasas y se restringen al mediterráneo occidental, principalmente a Sicilia (JiménezMejías \& Luceño, 2011), y de forma puntual en el este y suroeste de la Península Ibérica (Peña et al., 2003; Castroviejo, 2006, 2007; Sánchez-Gullón et al., 2017; Verloove, 2017).

En esta ocasión, damos a conocer una nueva localidad en el noroeste de la Península Ibérica:

Cyperus papyrus L., Sp. PI. 1: 47 (1753)

ESPAÑA. A Coruña: Arteixo, embalse de Rosadoiro, $43^{\circ} 9^{\prime} 26,61^{\prime \prime} \mathrm{N}$; $\left.8^{\circ} 29^{\prime} 54,83^{\prime \prime} \mathrm{O}\right], 15$ $\mathrm{m}, 08 / 05 / 2018, R$. Carballeira \& M. Souto (SANT 75740).

La población ocupa un área de $22,6 \mathrm{~m}^{2}$ con hojas de hasta 3-4 m de altura (Fig. 1), sin poder determinar el número de individuos debido al carácter rizomatoso de la especie. Se asienta sobre una zona fangosa en la orilla del embalse de Rosadoiro sometida a ciertas fluctuaciones estacionales del nivel de agua. El embalse se sitúa en una zona litoral en los límites de la costa Ártabra, un área muy irregular delimitada por colinas de entre 100 y 200 m de altitud, marcada por la influencia oceánica, que suaviza el clima con temperaturas medias mayores y períodos de heladas más cortos (Carballeira et al.,1983). En el embalse de Rosadoiro, además es conocida la presencia puntual de otras especies de plantas alóctonas como Tradescantia fluminensis Vell. en las zonas más antropizadas, e incluso es abundante la presencia de fauna acuática invasora, como Carassius auratus (Linnaeus, 1758) y Procambarus clarkii (Girard, 1852).

La primera referencia a una población naturalizada del papiro en la Península se debió a Guinea (1953), quien la citó de un pequeño humedal artificial de Cantabria, también en la región biogeográfica atlántica. Sin embargo, se ha considerado errónea tras el estudio del pliego correspondiente (MA 164585) por Laínz (1976). En el área mediterránea ibérica, la presencia de poblaciones naturalizadas de $C$. papyrus es más reciente, y se localiza en zonas adyacentes a la Albufera de Valencia (Peña et al., 2003; Sanz-Elorza et al., 2011) y del Delta del Ebro (Royo-Pla, 2006), en grandes acequias de riego y de arrozales, así como en la costa atlántica del suroeste ibérico en 


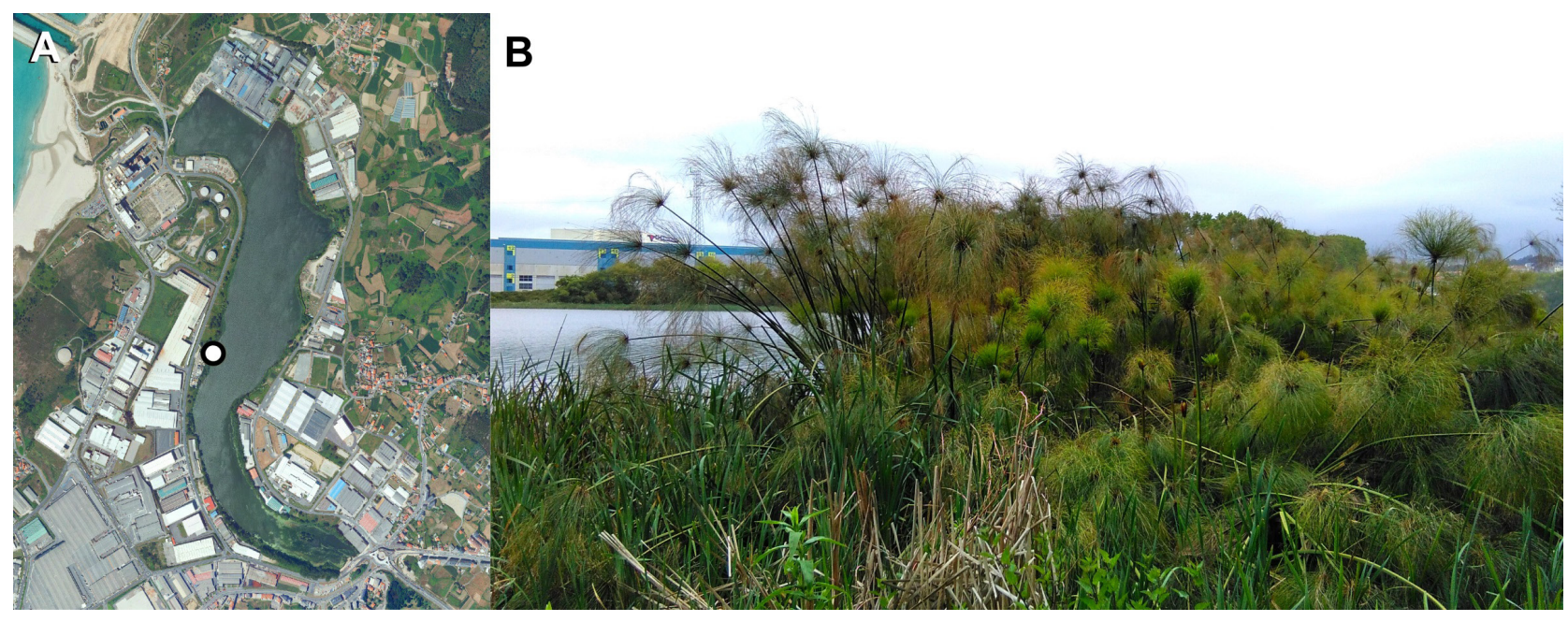

Figura 1. Fotografía aérea (PNOA 2017 @CNIG) de la situación (círculo blanco) de la nueva cita de Cyperus papyrus L. (A) y de la planta (B) en el embalse de Rosadoiro.

Figure 1. Aerial photograph (PNOA 2017 CCNIG) with the situation (white circle) of the new record of Cyperus papyrus $L$. (A), and of the plant $(B)$ in the Rosadoiro reservoir.

el entorno de las marismas de la Alquería, donde desemboca el río Tinto (Sánchez-Gullón et al., 2017). De todo ello se deduce que la distribución de las poblaciones ibéricas naturalizadas parece ligada a humedales litorales, con cierto grado de transformación antrópica, existiendo además una presencia puntual en las islas macaronésicas de Madeira (Vieira, 2002) y Tenerife (Verloove, 2017) (Fig. 2).

El área nativa de C.papyrus comprende climas subtropicales y tropicales, pero también zonas de clima mediterráneo, como la costa egipcia y el delta del río Nilo, por lo que existen semejanzas climáticas con el sur de Europa, que permiten comprender el asentamiento de poblaciones de esta especie. Así, los datos sobre su ecología en el delta del río Nilo indican que las temperaturas bajas ejercen un efecto negativo sobre el desarrollo de la especie (Serag, 2003) lo que puede ser un factor determinante en su asentamiento y distribución en Europa; así la ausencia de poblaciones en el interior peninsular puede deberse al efecto de la continentalidad. La presencia de esta especie en las islas macaronésicas puede explicarse por la latitud y las semejanzas de su clima subtropical con el existente en su área nativa en África continental.

La importancia cultural de $C$. papyrus desde la antigüedad y su presencia en la Europa mediterránea a lo largo de la historia, ha incrementado las posibilidades de eventos fundacionales y la estabilización de poblaciones en las áreas geográficas con mayor afinidad ecológica. En el curso del río Ciane, que desemboca cerca de Siracusa en Sicilia (Orti, 1834), existe una población muy antigua que actualmente constituye un espacio natural protegido por su interés histórico-cultural (EUAP, 2010). Esta situación conllevó a que algunos autores dudasen sobre el origen de su presencia en Europa y la incluyesen entre las especies autóctonas de Sicilia (Conti et al., 2005; Giardina et al., 2007; Brullo et al., 2011). Sin embargo, la interpretación más adecuada, como destacan Verloove et al. (2014), es que se trata de un arqueófito, lo que ha provocado su eliminación en los listados más recientes sobre la flora autóctona de la isla (Bartolucci et al., 2018). En la Península Ibérica, Castroviejo (2006, 2007) explica la presencia de las poblaciones como resultado de la dispersión de individuos de zonas de cultivo cercanas, como sugieren algunos pliegos de herbario de finales del s. XIX procedentes de las Islas Canarias (jardín de aclimatación de cultivos, La Paz, Tenerife, OHN 35202 y 35203), de comienzos del $\mathrm{s}$. XX de la Península Ibérica (estanques de los parques de Barcelona, MA 15743; ciudad de Almuñécar, Granada, MA 157556), y de la actualidad (zona ajardinada de la Acequia de Vir, Grao de Gandía, Valencia, BC 932621). Por otro lado, la distribución del papiro se circunscribe a zonas de influencia antrópica, y no existe constancia de que se comporte como una especie invasora ni en la Península Ibérica ni en Europa, de hecho, en el caso de Sicilia pese a tener una población estable muy antigua, su área de extensión se limita a la zona de asentamiento original. 


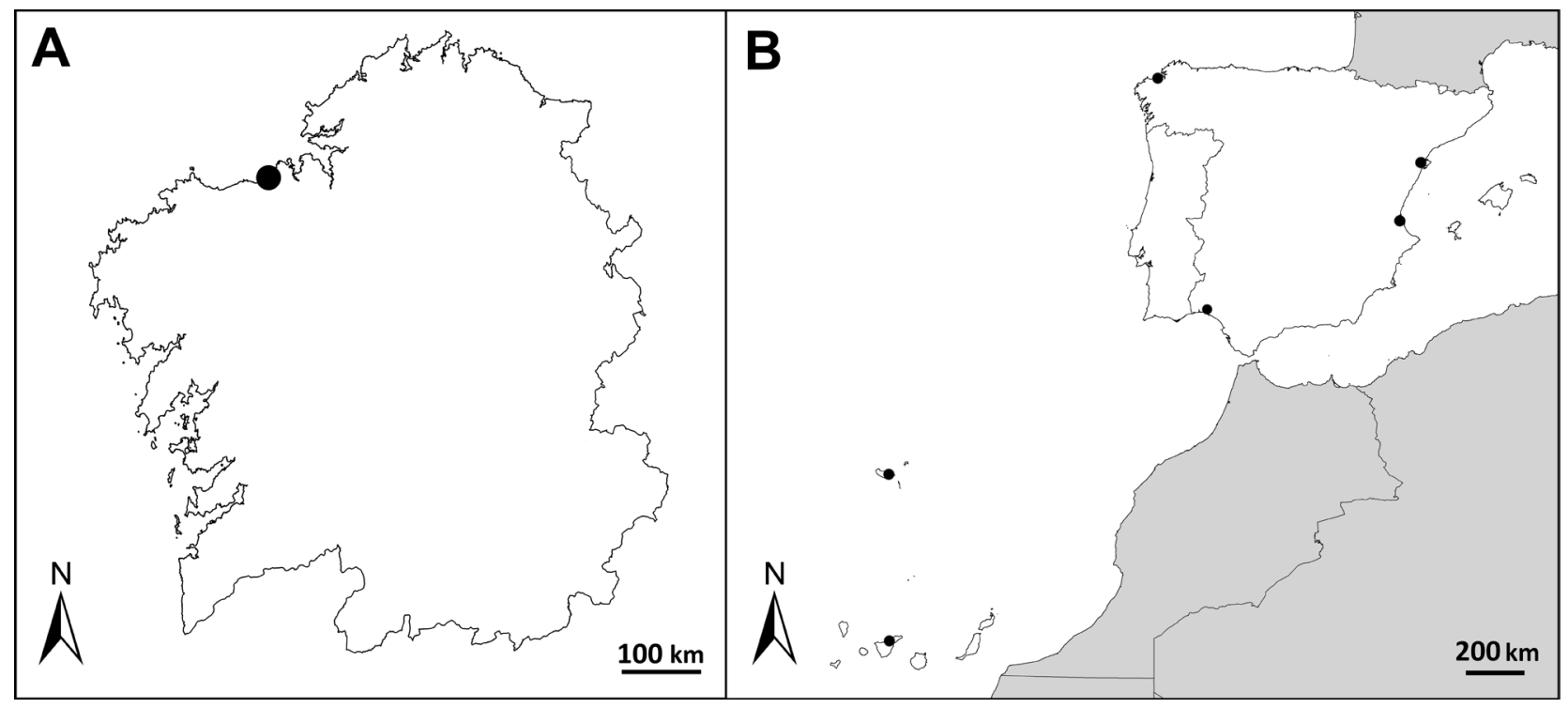

Figura 2. Distribución de las poblaciones naturalizadas de Cyperus papyrus L. en Galicia (A) y en la Península Ibérica, Madeira y Tenerife (B).

Figure 2. Distribution of the naturalized populations of Cyperus papyrus L. in Galicia (A), and in the Iberian Peninsula, Madeira and Tenerife (B).

\section{Agradecimientos}

Agradecemos las sugerencias recibidas por los revisores y el editor, Dr. Antonio Galán de Mera, que han mejorado considerablemente este trabajo.

\section{Bibliografía}

Allen, K.W. (1996). Papyrus some ancient problems in bonding. International Journal of Adhesion and Adhesives, 16(1), 47-51. doi: 10.1016/01437496(96)88486-7

Bartolucci, F., Peruzzi, L., Galasso, G., Albano, A., Alessandrini, A., Ardenghi, N.M.G., Astuti, G., Bacchetta, G., Ballelli, S., Banfi, E., et al. (2018). An updated checklist of the vascular flora native to Italy. Plant Biosystems, 152(2), 179-303. doi: 10.1080/11263504.2017.1419996

Beentje, H.J. (2017). Cyperus papyrus, Papyrus Sedge. The IUCN Red List of Threatened Species (fecha de consulta 10/09/2018). doi: 10.2305/IUCN.UK.2017-1. RLTS.T164158A66891399.en

Brullo, C., Minissale, P., Sciandrello, S. \& Spampinato, G. (2011). Phytogeographic survey on the endemic vascular flora of the Hyblaean territory (SE Sicily, Italy). Acta Botanica Gallica, 158(4), 617-631. doi: 10.1080/12538078.2011.10516299)

Carballeira, A., Devesa, C., Rubén Retuerto, R., Santillán, E. \& Ucieda, F. (1983). Bioclimatología de Galicia. A Coruña: Fundación Pedro Barrié de la Maza.

Castroviejo, S. (2006). Más sobre Cyperus y Kylinga en la Península Ibérica. Acta Botanica Malacitana, 31, 232-238. doi: 10.1080/00837792.2013.801110

Castroviejo, S. (2007). Cyperus. In S. Castroviejo, M.
Luceño, A. Galán, P. Jiménez Mejías, F. Cabezas \& L. Medina (Eds.). Flora iberica Vol. 18. CyperaceaePontederiaceae (pp. 8-27), Madrid: Consejo Superior de Investigaciones Científicas.

Conti, F., Abbate, G., Alessandrini, A. \& Blasi, C. (2005). An annotated checklist of the Italian vascular flora. Rome: Palombi Editori.

EUAP (2010). Elenco ufficiale delle aree protette, $6^{\circ}$ Aggiornamento approvato il 27 aprile 2010 e pubblicato nel Supplemento ordinario n. 115 alla Gazzetta Ufficiale n. 125 del 31 maggio 2010 (fecha de consulta 10/09/2018). Roma: Ministero dell'Ambiente. http:// www.gazzettaufficiale.it.

Giardina, G., Raimondo, F.M. \& Spadaro, V. (2007). A catalogue of plants growing in Sicily. Bocconea, 20, 5-582. doi: 10.1080/12538078.2011.10516299

Guinea, E. (1953). Geografía botánica de Santander. Santander: Imprenta Provincial de Santander, Diputación de Santander.

Jiménez-Mejías, P. \& Luceño, M. (2011). Cyperaceae. In Euro+Med PlantBase. The information resource for Euro-Mediterranean plant diversity (fecha de consulta 10/09/2018). Berlin: Botanic Garden and Botanical Museum Berlin-Dahlem. http://ww2.bgbm. org/EuroPlusMed/query.asp.

Kipkemboi, J. \& van Dam, A.A. (2016). Papyrus Wetlands. In C. Finlayson, G. Milton, R. Prentice \& N. Davidson (Eds.), The Wetland Book (pp. 1-15). Dordrecht: Springer. doi: 10.1007/978-94-007-6173-5_218-1

Laínz, M. (1976). Aportaciones al conocimiento de la Flora Cántabro-Astur, XI. Boletín del Instituto de Estudios Asturianos, 22, 3-44.

Orti, G.G. (1834). Al barone Vincenzo Mortillaro diretore del Poligrafo e conservatore del museo pubblico di Verona, parte 2, XI. Giornale di Scienze lettere e arti 
per la Sicilia, 47, 316-326.

Peña, C., Sebastián, A. \& Laguna, E. (2003). Cyperus papyrus L. en la Albufera de Valencia. Flora Montiberica, 23, 44-45.

Ramírez, P., Díaz Espinosa, A. \& Díaz, J.E. (2012). Cyperus papyrus. In A.M. Díaz-Espinosa, J.E. DíazTriana \& O. Vargas (Eds.), Catálogo de plantas invasoras de los humedales de Bogotá (pp. 106-111). Bogotá: Universidad Nacional de Colombia.

Royo Pla, F. (2006). Flora $i$ vegetació de les planes $i$ serres litorals compreses entre el riu Ebro i la serra d'Irta. Tesis doctoral. Barcelona: Universidad de Barcelona.

Sánchez Gullón, E., Verloove, F. \& Silva, V. (2017). New records of interesting xenophytes in the Iberian Peninsula. VII. Folia Botanica Extremadurensis, 11, 39-50. doi: 10.5209/rev_LAZA.2015.v36.48752

Sanz-Elorza, M., Guillot-Ortiz, D. \& Deltoro, V. (2011). La flora alóctona de la Comunidad Valenciana (España).
Botanica Complutensis, 35, 97-130. doi: 10.5209/rev_ BOCM.2011.v35.10

Serag, M.S. (2003). Ecology and biomass production of Cyperus papyrus L. on the Nile bank at Damietta, Egypt. Journal of Mediterranean Ecology, 4(3-4), 1524.

Verloove, F. (2014). A conspectus of Cyperus s.l. (Cyperaceae) in Europe (incl. Azores, Madeira and Canary Islands), with emphasis on non-native naturalized species. Webbia, 69(2), 179-223. doi: 10.1080/00837792.2014.975013

Verloove, F. (2017). New xenophytes from the Canary Islands (Gran Canaria and Tenerife; Spain). Acta Botanica Croatica, 76 (2), 120-131. doi: 10.1515/ botcro-2017-0013

Vieira, S. (2002). Cyperus papyrus L. DAISIE European Invasive Alien Species Gateway (fecha de consulta 10/09/2018). http//www.europe-aliens.org/ speciesFactsheet. 